\title{
Follicular Variant of Papillary Thyroid Carcinoma on the Basis of His- topathological and Immunohistochemical Diagnosis
}

\author{
Rahman $\mathrm{MM}^{1}$, Banu $\mathrm{SG}^{2}$, Barua $\mathrm{AR}^{2}$, Kamal M${ }^{2}$, Baqui $\mathrm{MN}^{3}$, Parveen $\mathrm{S}^{4}$ \\ ${ }^{I}$ Department of Cytopathology, National Institute of Cancer Research and Hospital, Dhaka, Bangladesh \\ ${ }^{2}$ Department of Pathology, Bangabandhu Sheikh Mujib Medical University, Dhaka, Bangladesh \\ ${ }^{3}$ Department of Pathology, Uttara Adhunik Medical College, Dhaka, Bangladesh \\ ${ }^{4}$ National Institute of ENT, Dhaka, Bangladesh \\ e-mail: mohammadmosiur76@gmail.com
}

\begin{abstract}
A significant increase in incidence of papillary thyroid carcinoma (PTC) has been noticed in recent decades worldwide. This is due to advances in medical surveillance, increased use of ancillary tests, and a minor component due to over diagnosis of PTC. Follicular variant of papillary thyroid carcinoma (FVPTC) is the second most common variant of PTC and comprises about $23-41 \%$. It is difficult to diagnose histologically when the distinctive nuclear features are either not well developed or present focally within the lesion. Several immunohistochemical markers (CD56, HMCK, GAL3, HBME-1 and CK19) either alone or combined in panels can be used to improve diagnostic accuracy. This study was aimed to differentiate FVPTC from other follicular patterned lesion of thyroid by histopathology and immunohistochemistry (IHC). A total of 50 histologically diagnosed cases of thyroid neoplasm were studied. The neoplastic cases included 40 cases of follicular variant papillary carcinoma (FVPC), 04 classic papillary carcinoma (PTC), 04 follicular carcinoma and 02 follicular adenoma. All cases were evaluated by IHC for the expression of CD56 and CK19 antibody. In case of FVPTC (n=40), 21 cases (52.5\%) were CK19 positive and CD56 negative as expected. Both markers were found positive in $06(15 \%)$ cases and CD56+ alone was found positive in $11(27.5 \%)$ cases of FVPTC, and a finding that goes against the diagnosis of FVPTC. The histopathological slides of these cases were reviewed and findings were recorded. All cases $(n=4)$ of classic PTC were CK19 positive and 03 (75\%) cases were found CD56 negative. Diagnoses of thyroid follicular lesions are primarily based on histological and cytomorphological criteria. However, there was a subset of follicular patterned tumors like FVPTC which lack unequivocal features of malignancy. Immunohistochemistry can improve diagnostic accuracy but needs additional studies for controversial cases. It may be considered these lesions as differentiated tumor of uncertain malignant potential (WDT-UMP) to avoid the using term carcinoma. Additional studies are needed for establishing more precise morphologic criteria and for identifying useful markers for differentiating benign from borderline or malignant thyroid lesions.
\end{abstract}

Keywords: Immunohistochemistry (IHC), Classic PTC, FVPTC, CD56, CK19, WDT-UMP etc.

\section{Introduction}

Thyroid cancer represents $1.5 \%$ of all cancers. ${ }^{1}$ Butit is the commonest endocrine cancer accounting for $92 \% .^{2}$ The annual incidence of thyroid cancer varies from 0.5 to 10 per 100,000 populations worldwide. Exact incidence of thyroid cancer in Bangladesh is not known. One study at the Institute of Nuclear Medicine and Thyroid Clinic in the then Institute of Post Grad- uate Medicine and Research (IPGMR), Dhaka reviewed 2629 thyroid patients from January 1994 to June 1995, and found thyroid carcinoma in $2.58 \%$ of patients. ${ }^{3}$

Studies on histopathological features revealed $85-90 \%$ of their study cases were papillary thyroid carcinoma (PTC) and 10-15\% were follicular carcinoma. ${ }^{4}$ Incidence of these two types of carcinoma increased in many places around the 
world over the past three decades..$^{5}$ This is due to advances in medical surveillance of impalpable nodules and increased emphasis on ancillary tests. ${ }^{6}$ Such increase also reflects a minor component of over diagnosis of PTC. It is clear that some cases do raise controversy as being PTC or non PTC. ${ }^{7}$

The most common subgroups of PTC are conventional papillary carcinoma (55-60\%) and follicular variant papillary thyroid carcinoma (FVPTC, 23-41\%). ${ }^{8}$ The neoplasm had a follicular architectural pattern but the nuclear features were that of the conventional PTC. ${ }^{9}$ The recognition of the FVPTC posed many problems at different levels. Interobserver variation in the diagnosis of FVPTC has been demonstrated even by experienced pathologists. ${ }^{10}$

Clinically, FVPTC presents either as a single nodule or arise against a background of multinodular goiter. ${ }^{9}$ The main differential diagnoses include a broad range of other follicular lesions that range from benign hyperplasic nodule to follicular adenoma to follicular carcinoma. ${ }^{11}$ Diagnosis by fine-needle aspiration cytology and intraoperative frozen section examination are difficult and unreliable. Study has reported a very low sensitivity of FNA cytology for the identification of FVPTC and this experience has been largely paralleled with intraoperative frozen section examination. The majority of these tumors can only be reliably identified by examination of permanent sections from surgically resected lesions. ${ }^{11}$

Difficulties for diagnosis can also arise during the evaluation of permanent sections when the distinctive nuclear features are either not well developed or only present focally within the lesion. ${ }^{12}$ It was applied ancillary techniques, particularly immunohistochemistry and molecular techniques in an attempt to solve these problematic cases. ${ }^{7}$ Immunohistochemical markers like CD56, High Molecular Weight cytokeratin (HMCK), Galectine -3 (GAL3), Hector Battifora mesothelial cell 1 (HBME-1), Cytokeratin 19 (CK19) etc either alone or in combined panel can improve the diagnostic accuracy of follicularpattern thyroid lesions. ${ }^{10}$ It is a type I intermediate filament protein and the smallest known keratin. CK19 shows diffuse and strong cytoplasmic staining in papillary carcinoma including
FVPTC. ${ }^{13,15}$ CD56 (NCAM-Neural Cell Adhesion Molecule) is present normally in NK cells, activated $\mathrm{T}$ cells, large granular lymphocytes, specific endocrine cells, brain tissue and also follicular epithelial cells of normal thyroid. Loss of CD56 correlates with metastatic potentials and poor prognostic outcome in some malignancies. Strong and complete membranous expression with or without cytoplasmic staining qualified the case as positive for CD56. ${ }^{6,7}$ Low or absent expression of CD56 is noted in papillary carcinoma of thyroid. ${ }^{7}$

In the NCDB (National Cancer Data Base, USA) study, the 10 year relative survival rates for patients with papillary and follicular carcinoma were $93 \%$ and $85 \%$, respectively. ${ }^{14}$ If appropriately treated, papillary carcinoma has an excellent long-term prognosis. Appropriate treatment rests primarily on the ability of the pathologist to render an accurate diagnosis. ${ }^{15}$ If diagnosis can be made correctly, these individuals will be spared of unnecessary, aggressive surgical and radioactive iodine therapy, and morbidity and financial costs related to these procedures. ${ }^{16}$

The present study was aimed to differentiate follicular variant of papillary thyroid carcinoma from other follicular-patterned lesions of the thyroid by histopathological and immunohistochemical methods.

\section{Materials and Methods}

This cross-sectional study was conducted in the Department of Pathology, Bangabandhu Sheikh Mujib Medical University (BSMMU), Dhaka during the period of January 2011 to November 2012. The patients who underwent thyroid surgery for suspected malignant thyroid lesion were enrolled in the study.

A total of 40 cases of histologically diagnosed follicular variant of papillary thyroid carcinoma with proper clinical information from all ages and both sexes were included. Another 10 cases of follicular carcinoma $(n=4)$, follicular adenoma $(n=2)$ and classic papillary carcinoma $(n=4)$ were also included for comparison in immunohistochemical study to see the pattern of expression of used antibodies in these tumours. Cases were collected from the Departments of General Surgery and Head, Neck and ENT Surgery, 
BSMMU and other private hospitals in Dhaka city. Clinical information was recorded in preformed clinical proforma. Thyroid specimens received in $10 \%$ neutral buffered formalin were kept for overnight fixation after one $\mathrm{cm}$ slices. In the next morning the specimens were examined during gross cut up and representative tissue blocks were taken. Tissue processing was performed in automated tissue processor following standard protocol. For histomorphological examination, sections were stained with haematoxyllin and eosin (H and E) stain. Slides of all cases were examined and interpreted by experienced pathologists. Decision in controversial cases was made by consensus meeting at the multi-head microscope.

For immunohistochemical study, sections were taken from the paraffin blocks on HistoGrip coated slides. DAKO EnVision ${ }^{\mathrm{TM}+} / \mathrm{HRP}$ (Horseradish peroxidase) System which is based on advance Labeled Strept Avidin-Biotin (LSAB) method was used for visualizing the section. This method was carried out by DAKO autostainer. Two primary antibodies (CK19 and CD56) were used. Results of immunostains were assessed by light microscopy. CK19 shows diffuse and strong cytoplasmic staining in papillary carcinoma including FVPTC. Strong and complete membranous expression with or without cytoplasmic staining qualified as positive for CD56 (figure.1).
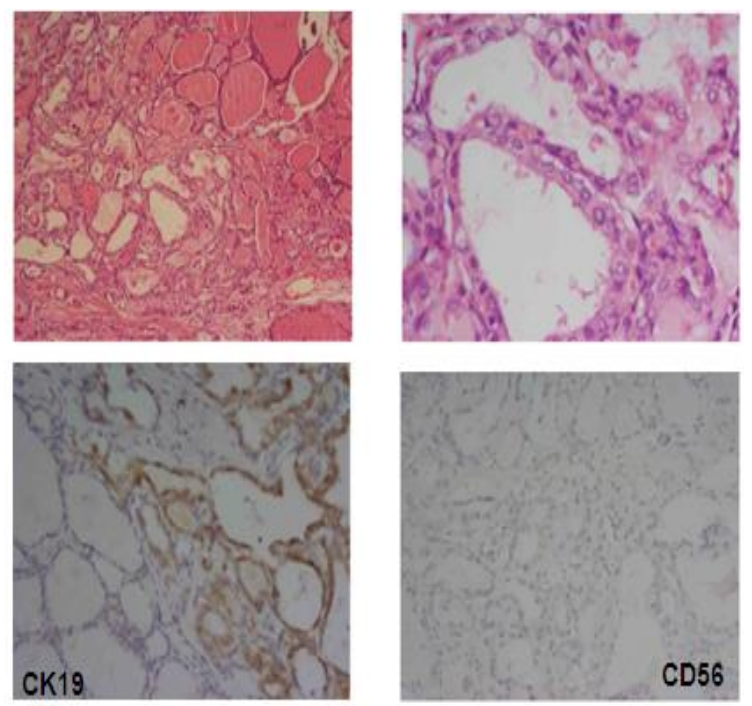

Figure 1: A case of FVPTC showing typical histopathological and immunostaining (CK19+ve and CD56-ve) pattern.

Multiple microscopic fields were examined, and the findings were expressed semi-quantitatively according to the estimated percentage of positive tumor cells: zero (0)- staining of $<10 \%$ of the cells; 1 - staining in $10-33 \%$ of the cells; 2 - staining in $33-66 \%$ of the cells; 3 - staining in $>66 \%$ of the cells. A score of zero was considered negative, and scores of 1-3 were considered positive.

Data were analyzed by standard statistical method and expressed as mean, standard deviation, ratios and percentages. Written informed consent was obtained from the patients/ patient's guardians Ethical clearance for the study was taken from the "Institutional Review Board (IRB), BSMMU.

\section{Results}

Size of the tumor ranged from $0.7 \mathrm{~cm}$ to $6.5 \mathrm{~cm}$ with a mean of $3.1 \pm 1.7 \mathrm{~cm}$ in cases of FVPTC. In PPTC and FC, mean tumor size was $3 \mathrm{~cm}$ and $3.87 \mathrm{~cm}$ respectively.

In FVPTC cases, 3(7.5\%) had capsular invasion and $1(2.5 \%)$ showed vascular invasion. Lymph node metastasis was seen in $1(2.5 \%)$ case. All cases of follicular carcinoma showed capsular invasion, while vascular invasion was seen in $2(50 \%)$ cases. Lymph node metastasis was not seen in follicular carcinoma and classic PTC.

Variations in follicular size were observed in 38 $(95.0 \%)$ cases of FVPTC. Follicles were found uniform in follicular adenoma and $3(75 \%)$ cases of follicular carcinoma. Cystic degeneration within the tumor nodule was found in $8(20 \%)$ cases of FVPTC, 2(50\%) cases of classic PTC, $1(50 \%)$ case of FA and $1(25 \%)$ case of FC. Psammoma bodies were present in one case of FVPTC and one case of classic PTC. Nonpsammomatous calcification was seen in $3(7.5 \%)$ cases of FVPTC.

Diffuse and uniform nuclear clearing were observed in $22(55 \%)$ cases of FVPTC and all cases of classic PTC. Nuclear clearing was found focal and mostly in the centre of the nodule in 18 (45\%) cases. 38 (95\%) cases of FVPTC and all cases of classic PTC showed intranuclear grooves. Four (10\%) cases of FVPTC and all cases of classic PTC showed intranuclear pseudoinclusions in many cells. Nuclear pseudoinclusions were present in few cells of 29 $(72.5 \%)$ cases and were absent in $7(17.5 \%)$ cases. 
CK 19 showed positive expression in papillary carcinoma and weak to absent expression in non PTC lesions. In this study, CK19 positive expression accounted for 27(67.5\%) cases of FVPTC and all cases of classic PTC. FCs and FAs showed variable CK19 expression, which was completely negative (in cases of FA) to weakly positive (25\% cases of FC). The sensitivity and specificity of CK19 in papillary carcinoma (including Classic PTC and FVPTC) were $70.4 \%$ and $83.3 \%$ respectively.

CD56 negative expression accounted for 23(57.5\%) of cases of FVPTC and 3(75\%) cases of classic PTC. All FA and FC showed positive CD56 expression. The sensitivity and specificity of CD56 negative expression in papillary carcinoma (including classic PTC and FVPTC) are $59 \%$ and $83.3 \%$ respectively. When two antibodies were considered combindly, 21(52.5\%) cases of FVPTC were CK19 positive and CD56 negative as expected.

Table I: Combined immunoexpression of CK19 and CD56 in FVPTC

\begin{tabular}{ccc}
\hline Immunopattern & Frequency & Percent \\
\hline CK19+ CD56- & 21 & 52.5 \\
CK19- CD56+ & 11 & 27.5 \\
CK19+ CD56+ & 6 & 15.0 \\
CK19- CD56- & 2 & 5.0 \\
Total & $\mathbf{4 0}$ & $\mathbf{1 0 0 . 0}$ \\
\hline
\end{tabular}

Both markers were found positive in $6(15 \%)$ cases, only CD56 positivity was found in $11(27.5 \%)$ cases and both markers were found negative in 2(5\%) cases of FVPTC. In classic PTC, 03 (75\%) cases showed CK19 positive and CD56 negative immunoexpression. All FA cases were CD56 positive and CK19 negative (figure 2). Three $(75 \%)$ cases of FC showed CD56 positive and CK19 negative immunoexpression.
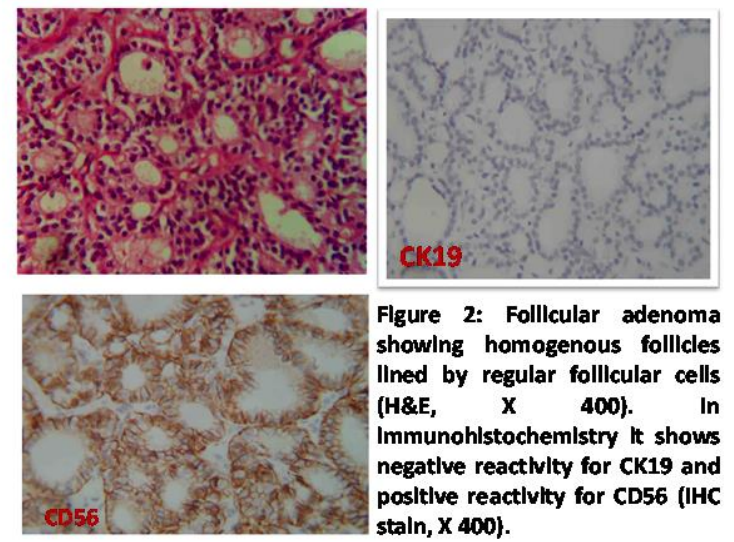

Flgure 2: Folllcular adenoma showing homogenous follicles IIned by regular follicular cells (H\&E, $X$ 400). In Immunohlstochemlstry it shows negatlve reactivity for CK19 and positive reactlvity for CD56 (IHC stain, X 400).

\section{Discussion}

The standard procedure of diagnosing thyroid lesions is histological examination of routine $\mathrm{H}$ and $\mathrm{E}$ stained sections. FVPTC often poses a trouble to be distinguished from other follicular patterned thyroid lesions when the tumor is encapsulated and nuclear features are present focally or in multiple microscopic foci. Immunohistochemical markers have been found effective in distinguishing PTC including follicular variants from other follicular lesions. In the present study, IHC study (CK19 and CD56 markers) was carried out on histologically diagnosed thyroid tumours.

The mean age of the 40 cases of FVPTC was $37.8 \pm 13.3$ years. Mean age of classic PTC was 35.55 years. The study showed a male to female ratio of 1:3 in FVPTC. Similar findings were found by Bose et al ${ }^{18}$, Yuksel et al ${ }^{8}$ and Mazzaferri and Masoll 2002..$^{5}$ In cases of FC, mean age was 52.5 years which was similar as described by Simoes et al.. ${ }^{19}$ Grossly the tumors were found encapsulated in $12(30 \%)$ cases and non-encapsulated in 28 (70\%) cases. Baloch and LiVolsi ${ }^{20}$ stated that some FVPTCs were shown to be completely encapsulated whereas others had partial to total lack of capsule. Among the nuclear features, nuclear clearing was observed diffusely and uniformly distributed in $22(55 \%)$ cases of FVPTC. In 18 (45\%) cases nuclear clearing was found focal and mostly in the centre of the nodules. Wenig ${ }^{21}$ found intranuclear pseudoinclusions in $25 \%$ cases and abundant nuclear grooves in $100 \%$ cases of papillary carcinoma. In this study, 38 (95\%) cases of FVPTC showed intranuclear grooves. Intranuclear pseudoinclusions were observed in many cells of $4(10 \%)$ cases of FVPTC; however, these features were observed in few cells of $29(72.5 \%)$ cases and were absent in 7 (17.5\%) cases.

Papillary carcinoma (including FVPTC) showed positive reactivity of CK19 in 27 cases and thesensitivity was $70 \%$. In a study by Bose et al $2012^{18}$ it was $77 \%$. In other study by Cheung et 
al. $2001^{14}$ sensitivity was $85 \%$ for classic PTC and 57\% for FVPTC.

Table II: Pattern of expression of CK19 and CD56 in defferent types of thyroid neoplasm (PTC, FVPTC, FA and FC) by different studies

\begin{tabular}{|c|c|c|c|c|c|c|c|c|c|}
\hline \multirow[t]{2}{*}{ Investigators } & \multicolumn{3}{|c|}{$\begin{array}{l}\text { PTC including } \\
\text { FVPTC }\end{array}$} & \multicolumn{3}{|c|}{ FA } & \multicolumn{3}{|c|}{ FC } \\
\hline & $\begin{array}{l}\text { No. of } \\
\text { cases }\end{array}$ & CK19+ & CD56- & $\begin{array}{l}\text { No. of } \\
\text { cases }\end{array}$ & CK19+ & CD56+ & $\begin{array}{l}\text { No. of } \\
\text { cases }\end{array}$ & CK19+ & CD56+ \\
\hline $\begin{array}{l}\text { Demellaway et } \\
\text { al. } 2008\end{array}$ & $\begin{array}{l}72 \% \\
23)\end{array} \quad$ FVPTC $=$ & $85 \%$ & $100 \%$ & 32 & $26 \%$ & $100 \%$ & 02 & $0 \%$ & $100 \%$ \\
\hline Park et al.2009 & 67 & $98.5 \%$ & $92.5 \%$ & 15 & $50 \%$ & $72.6 \%$ & 23 & Varia-ble & $95.6 \%$ \\
\hline Saleh et al.2010 & 32(FVPTC=12) & $90 \%$ & -- & 46 & $50 \%$ & -- & 22 & $86.3 \%$ & -- \\
\hline Shin et al.2011 & 80(FVPTC=02) & $100 \%$ & $95 \%$ & 05 & $80 \%$ & $100 \%$ & -- & -- & -- \\
\hline $\begin{array}{l}\text { Cheung et al. } \\
2001\end{array}$ & $\begin{array}{l}\text { 138 } \\
84)\end{array} \quad$ FVPTC $=$ & $\begin{array}{lr}85 \% & \text { (classic } \\
\text { PTC) } & 57 \% \\
\text { (FVPTC) } & \end{array}$ & -- & 35 & $\begin{array}{l}\text { Some Show } \\
\text { Focal positivi- } \\
\text { ty }\end{array}$ & -- & 04 & $0 \%$ & -- \\
\hline Bose et al.2012 & 22 & $77 \%$ & $100 \%$ & 08 & $75 \%$ & -- & -- & -- & -- \\
\hline In present study & $(\mathrm{FVPTC}=$ & $\begin{array}{c}70 \% \text { (classic } \\
\text { PTC }=100 \% \& \\
\text { FVPTC }=67.5 \% \text { ) }\end{array}$ & $\begin{array}{c}59 \%(\text { classic } \\
\text { PTC }=75 \% \& \\
\text { FVPTC }=57.5 \\
\%)\end{array}$ & 02 & $0 \%$ & $100 \%$ & 04 & $25 \%$ & $75 \%$ \\
\hline
\end{tabular}

CD56 negative expression was found in 26 cases of papillary carcinoma (including FVPTC) and the sensitivity of CD56 was 59\%. Different studies done by Demellawy al. $2008^{7}$, Shin et al. $2011^{13}$ and Park et al. $2009^{6}$ showed significant negative CD56 expression (100\%, $72.7 \%$ and $92.5 \%$ respectively) in papillary carcinoma including FVPTC (Figure 3).

Immunohistochemistry showed some differences in staining reactions from other studies. Twenty three (out of 44) cases of papillary carcinoma (including FVPTC) were CK19 positive and CD56 negative as expected. The sensitivity was $54.4 \%$ when used combindly. The differences might be due to factors like antibodies used, dilution and antigen retrieval methods, type of tissue fixative used, time of fixation and histological types of tumor.

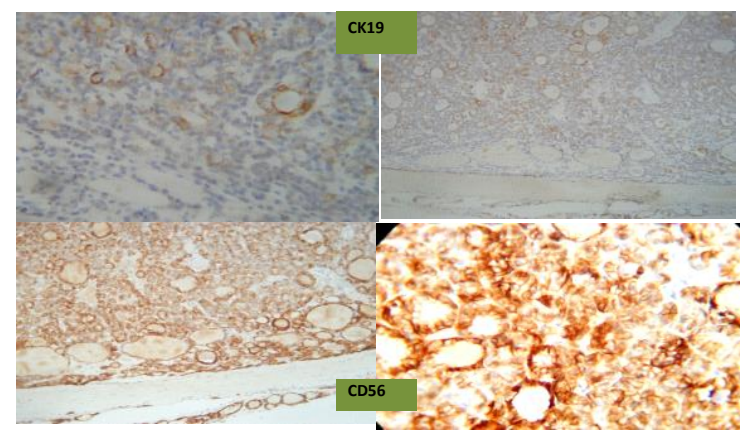

Figure 3: Immunopattern of this case was not in favour of FVPTC (CK19+ve and CD56+ve) which was histologically diagnosed as FVPTC

Most of the tumors (40 out of 50 cases) in the present study were FVPTC but in other studies, major group was classic PTC. The interobserver variability in the diagnosis of FVPTC is well recognized, and in current study there may be some cases that were incorrectly diagnosed during histological examination.

Chernobyl Pathologists Group ${ }^{12}$ introduced a new category of tumors which they termed as well differentiated tumors of uncertain malignant potential (WDT-UMP). They defined 'WDTUMP' as lesions with questionable nuclear changes and questionable or absent capsular invasion. This new terminology WDT-UMP reflects a borderline category which adds to the existing trouble significantly. ${ }^{17}$
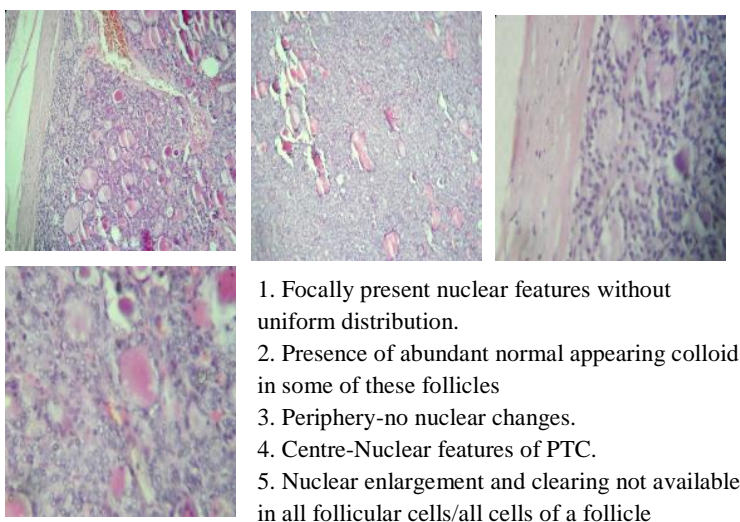

1. Focally present nuclear features without uniform distribution.

2. Presence of abundant normal appearing colloid in some of these follicles

3. Periphery-no nuclear changes.

4. Centre-Nuclear features of PTC.

5. Nuclear enlargement and clearing not available in all follicular cells/all cells of a follicle

Figure 4: $\mathrm{H}$ and $\mathrm{E}$ stained slides of a case where immunohistochemical result was not in favour of FVPTC (in figure. 3) and histopathological slides were reviewed and findings were recorded.

In this study, it was reviewed the $\mathrm{H}$ and $\mathrm{E}$ slides of cases (Figure 4) in which immunohistochemistry findings were not in favour of FVPTC. These tumors actually remained in the grey zone 
(whether FVPTC or not) by histomorphological study. Possibly it is better to group these tumors as WDT-UMP. Kakudo et al. $2011^{22}$ found that $1.1 \%$ tumor of their study cases of thyroid specimen were actually to be categorized as WDTUMP. Similar findings were seen in by Hofman et al $^{23}(1.5 \%)$ and by Piana et al ${ }^{24}(0.5 \%)$.

\section{Conclusion}

The distinction of the borderline lesion from FVPTC is not simply a matter of tumor classification but also meaningful on clinical ground. Accurate diagnosis is very critical for postoperative management of patients. WDT-UMP had a favorable outcome. Incorrect interpretation can cause the patients significant psychological and social problems, and unnecessary increase in healthcare cost.

Nonetheless, there are still questions to answer and additional studies (i.e. molecular) are needed for establishing more precise qualitative and quantitative morphologic criteria and for identifying useful markers for differentiating benign from borderline or malignant thyroid lesions.

Acknowledgement: The study was partly supported by a grant from Bangladesh Medical Research Council (BMRC). We express our gratitude to BMRC for the support.

\section{References}

1. Choudhury AM, Shaikh AA. Management of papillary and follicular (differentiated) thyroid carcinoma-an update. Bangladesh J Otorhinolaryngology. 2010; 16 (2): 126-30.

2. Niazi S, Arshad M, Muneer M. A histopathological audit of thyroid surgical specimens. Annals King Edward Med Coll. 2007; 13: 51-56.

3. Alauddin M, Joarder AH. Management of thyroid carcinoma. The ORION Medical Journal. 2004; 18 : 163-66.

4. Albores SJ, Gould E, Vardaman C, Vuitch F. The macro follicular variant of papillary thyroid carcinoma: a study of 17 cases. Hum Pathol. 1.991; 22: 1195-1205.

5. Mazzaferri EL, Massoll N. Management of papillary and follicular (differentiated) thyroid carcinoma: new paradigms using recombinant human thyroglobulin. Endocrine Related Cancer. 2002; 9 (4): 227-47.

6. Park WY, Jeong SM, Lee JH, Kang JH ,Sin DH , Choi KU et al. Diagnostic value of decreased ex- pression of CD56 protein in papillary carcinoma of the thyroid gland. Basic and Applied Pathology. 2009; 2: 63-68.

7. Demellawy DE, Nasr A, Alowami S. Application of CD56, P63 and CK19 immunohistochemistry in the diagnosis of papillary carcinoma of the thyroid. Diagnostic Pathology. 2008; 35: 1-12.

8. Yuksel O, Kurukahvecioglu O, Ege B, Ekinci O, Aydin A, Poyraz A et al. The relation between pure papillary and follicular variant in papillary thyroid carcinoma. Endocrine Regulations. 2008; 42: 29-33.

9. Salajegheh A, Petcu EB, Smith RA, Lam AK. Follicular variant of papillary thyroid carcinoma: a diagnostic challenge for clinicians and pathologists. Post grad Med J. 2008; 84: 78-82.

10. Fischer S, Asa SL. Application of Immunohistochemistry to Thyroid Neoplasm. Arch Pathol Lab Med. 2008; 132: 359-72.

11. Suster S. Thyroid Tumors with Follicular Growth Pattern Problems in Differential Diagnosis. Arch Pathol Lab Med. 2006; 130: 984-88.

12. Williams ED. Guest editorial: two proposals regarding the terminology of thyroid tumors. Int J Surg Pathol. 2000; 8: 181-83.

13. Shin MK, Kim WK, Ju YS. CD56 and High Molecular Weight Cytokeratin as Diagnostic Markers of Papillary Thyroid Carcinoma. The Korean Journal of Pathology. 2011; 45: 477-84.

14. Cheung C C, Ezzat S, Freeman J L, Rosen I B, Asa $\mathrm{S}$ L. Immunohistochemical Diagnosis of Papillary Thyroid Carcinoma. Modern Pathology. 2001; 14 (4): 338-42.

15. NCCN (National Comprehensive Cancer Network), Clinical Practice Guidelines in Oncology. 2002; Thyroid Carcinoma. NCCN, Washington, USA.

16. Rivera M, Ricarte J, Knauf J, Shaha A, Tuttle M, Fagin JA et al. Molecular genotyping of papillary thyroid carcinoma follicular variant according to its histological subtypes (encapsulated vs. infiltrative) reveals distinct BRAF and RAS mutation patterns. Modern Pathology. 2010; 23: 1191-1200.

17. Bukhari U, Sadiq S, Kehar SI. Differential Expression of CK 19 in follicular adenoma, Welldifferentiated tumour of uncertain malignant potential (WDT-UMP) and follicular variant of papillary carcinoma. J Pak Med Assoc. 2009; 59: 15-18.

18. Bose D, Das RN, Chatterjee U, Banerjee U. Cytokeratin 19 immunoreactivity in the diagnosis of papillary carcinoma of thyroid. Indian Journal of Medical and Paediatric Oncology. 2012; 33: 107-11. 
19. Simoes MS, Asa SL, Kroll TG, Nikiforov Y, Delellis R. Follicular carcinoma. In: Delellis RA, Lioyd RV, Heitz PU, Eng C (editors). World Health Organization classification of tumour, Pathology and genetics of tumour of endocrine organs. 2004; 71-72.

20. Baloch ZW, LiVolsi VA. Our approach to follicularpatterned lesions of the thyroid. Journal of Clinical Pathology. 2007; 60: 244-50.

21. Wenig BM, Thyroid Papillary Carcinoma, Follicular (and Encapsulated) Variant [Internet]. In Online: USCAP virtual slide Box- surgical pathology [Cited 2012 Sep 27]. Available from: http://www.uscap.org /site /97th/specsurgh5v.htm
22. Kakundo K, Bai Y, Liu Z, Li Y, Ito Y, Ozaki T. Classification of thyroid follicular cell tumour: with special reference to borderline lesions. Endocrine Journal 2011; 11-18.

23. Hofman V, Lassalle S, Bonnetaud C, Butori C, Loubatier C, Bordone O. Thyroid tumours of uncertain malignant potential: frequency and diagnostic reproducibility. Virchows Arch. 2009; online publication: 1-13.

24. Piana S, Frasoldai A, Felice ED. Encapsulated well differentiated follicular patterned thyroid carcinoma do not play a significant role in the fatality rate from thyroid carcinoma. American Journal of Surgical Pathology 2010; 34: 868-72. 